\title{
Messung mechanischer Beanspruchungen in laufenden Schleifprozessen
}

\author{
Andreas Tausendfreund ${ }^{1}$, Gabriela Alexe ${ }^{1}$, Dirk Stöbener ${ }^{1,2}$, Andreas Fischer ${ }^{1,2}$ \\ ${ }^{1}$ Bremer Institut für Messtechnik, Automatisierung und Qualitätswissenschaft (BIMAQ), \\ Linzer Straße 13, 28359 Bremen, Deutschland \\ ${ }^{2}$ MAPEX Center for Materials and Processes, \\ Postfach 330 440,28334 Bremen, Deutschland
}

\section{Zusammenfassung}

Die Kenntnis der während eines Fertigungsprozesses (z.B. Schleifen) auftretenden Beanspruchungen und der im Material verbleibenden Veränderungen wird im Konzept der Prozesssignaturen genutzt, um den Fertigungsprozess zu optimieren und mit anderen zu vergleichen (z.B. Laserbearbeitung). Voraussetzung für die Erstellung einer Prozesssignatur ist, dass die Beanspruchungen während des laufenden Prozesses charakterisiert werden können. Aufgrund der rauen Prozessbedingungen gibt es beim Schleifen bisher keine prozessbegleitende Technik zur Messung der Beanspruchungen in Form von Verschiebungen und Dehnungen in der bearbeiteten Randzone. Aus diesem Grund wird die Eignung der Speckle-Fotografie für prozessbegleitende Messungen von Materialbeanspruchungen in einem laufenden Schleifprozess ohne Kühlschmierstoff nachgewiesen und ein Konzept für eine Messung unter dem Einsatz von Kühlschmierstoffen vorgestellt.

Keywords: In-Prozess-Messung, Speckle-Fotografie, Schleifprozess, Verformungs- und Dehnungsmessung

\section{Einleitung}

In der Metallverarbeitung geht es darum, aus einem Grundmaterial ein Produkt mit funktionalen Eigenschaften zu erzeugen. Hierfür sind in der Regel verschiedene Fertigungsprozesse erforderlich, um die Form, die Oberflächenrauheit bzw. die Randzoneneigenschaft des Werkstücks gezielt einzustellen. Eine Prozesskette wie z.B. das Fräsen mit anschließenden Schleifen und Laserhärten lässt sich unter Umständen kostengünstiger gestalten, indem nach dem Fräsprozess ein Schleifprozess mit kombiniertem Schleifverfestigen erfolgt. Aktuelle Forschungsanstrengungen im Rahmen des Sonderforschungsbereichs SFB/TRR 136 zielen deshalb darauf $a b$, Fertigungsschritte prozessunabhängig über die sogenannte Prozesssignatur zu beschreiben. Die Grundvoraussetzung dafür ist jedoch eine In-ProzessCharakterisierung der mechanischen Beanspruchungen des Werkstoffs in Form von Verformungen und Dehnungen.

Prädestiniert für die berührungslose Messung von In-Plane-Verschiebungsfeldern sind die seit den 1970er Jahren erforschten SpeckleKorrelations-Verfahren [1, 2]. Bei diesen Verfahren wird die zu messende Oberfläche mit einem Laser beleuchtet und die Auswertebereiche in den mit der Kamera aufgenommenen Specklebildern - jeweils vor und während des
Auftretens der Verformung - miteinander korreliert. Aus den korrelierten Bildausschnitten lassen sich lokale Ortsverschiebungen bestimmen, deren theoretische Auflösungen lediglich durch die Heisenbergsche Unschärferelation beschränkt sind [3]. Für Verschiebungsmessungen können mit aktueller Kamera- und Lasertechnologie Unsicherheiten von weniger als $7 \mathrm{~nm}$ erreicht werden [3]. Als berührungsloses optisches Messverfahren ist die SpeckleFotografie daher prädestiniert für schnelle und präzise In-Prozess-Messungen. Die SpeckleFotografie konnte kürzlich für Verformungsmessungen im Walz- [4] und Fräsprozess [5] eingesetzt werden. Aufgrund der rauen Umgebungsbedingungen mit starkem Funkenflug oder dem Einsatz von Kühlschmierstoffen ist die Speckle-Fotografie beim Schleifprozess unter realen Bedingungen jedoch noch nicht zur Anwendung gekommen.

Der vorliegende Beitrag beschäftigt sich deshalb mit speckle-fotografischen In-ProzessDehnungsmessungen beim Schleifen. Die sich hierbei ergebenden wissenschaftlichen Fragestellungen sind, ob trotz der anspruchsvollen Prozessbedingungen Speckle-Fotografie-Messungen möglich sind, wie eine robuste Bildauswertung erzielt werden kann und welche Messabweichungen sich durch den Einsatz von Kühlschmierstoffen ergeben. 


\section{Verschiebungs- und Dehnungsmessungen mittels Speckle-Fotografie}

Die Speckle-Fotografie ist eine bekannte Technik zur Charakterisierung von Verschiebungsfeldern in der Ebene einer zu messenden Oberfläche [1,2]. Eine Kamera erfasst hierbei das an der Oberfläche diffus gestreute kohärente Laserlicht. Die von der Oberfläche modulierten Phasenelemente der Lichtwelle erzeugen ein Bild der Topographie in der Bildebene (auf dem Kamerachip), welches aufgrund von konstruktiver und destruktiver Interferenz mit einem Pünktchen- bzw. dem Specklemuster überlagert ist (Abbildung 1). Die GröBe der Speckle kann durch die Öffnung der Blende in einem weiten Bereich variiert werden. Ein herausragendes Merkmal der subjektiven Speckle ist, dass sie - entsprechend dem Abbildungssystem - spezifischen Oberflächenpunkten zugeordnet werden können. Daher lassen sich Verschiebungen einzelner Speckle als Verschiebungen lokaler Oberflächenpunkte in der Objektebene interpretieren. Zur Berechnung des globalen Verschiebungsfelds der gemessenen Oberfläche, rastert ein kleines Auswertefenster über das Messfeld, hierbei wird in jedem Punkt jeweils die Kreuzkorrelation zwischen zwei aufeinanderfolgenden Specklebildern gebildet. Die Größe des Auswertefensters lässt sich automatisch berechnen und sollte mindestens drei Speckle auf der Diagonalen enthalten. Ein optimaler Speckledurchmesser beträgt nach Zhou und Goodson ca. 3-5 Pixel [6]. Durch die Bestimmung der Verschiebung der Korrelationsmaxima können elastische und plastische Verformungen mit einer räumlichen Auflösung von etwa $20 \mathrm{~nm}$ und zeitlichen Auflösung im Mikrosekundenbereich rekonstruiert werden [5].

Nach der Berechnung des Punktverschiebungsfeldes $\mathbf{P}(\mathrm{u}, \mathrm{v})$ (wobei $\mathrm{u}$ und $\mathrm{v}$ die Verschiebungen in $\mathrm{x}$ - und $\mathrm{y}$-Richtung sind) lässt sich das Gradientenfeld $\mathbf{F}_{2 \mathrm{D}}$ der Verformung nach Kajberg [7] bestimmen:

$$
\mathbf{F}_{2 \mathrm{D}}=\left(\begin{array}{cc}
1+\frac{\partial u}{\partial x} & 1+\frac{\partial u}{\partial y} \\
\frac{\partial v}{\partial x} & 1+\frac{\partial v}{\partial y}
\end{array}\right) .
$$

Die Polardarstellung des $\mathbf{F}_{2 \mathrm{D}}$ Felds führt $z u$

$$
\mathbf{F}_{2 D}=\mathbf{R}_{2 D} \mathbf{U}_{2 D},
$$

wobei $\mathbf{R}_{2 \mathrm{D}}$ der Rotationstensor $\left(\mathbf{R}^{\mathrm{T}} \mathrm{R}_{2 \mathrm{D}}=\mathrm{I}\right)$ und $\mathbf{U}_{2 D}$ der gesuchte positiv definite und symmetrische Dehnungstensor $\left(\mathbf{U}_{2 \mathrm{D}}=\mathbf{U}_{2 \mathrm{D}}\right)$ ist. Zunächst kann $\mathbf{F}_{2 \mathrm{D}} \mathbf{F}_{2 \mathrm{D}}=\left(\mathbf{R}_{2 \mathrm{D}} \mathbf{U}_{2 \mathrm{D}}\right)^{\mathrm{T}} \mathbf{R}_{2 \mathrm{D}} \mathbf{U}_{2 \mathrm{D}}=\mathbf{U}_{2 \mathrm{D}} \mathbf{U}_{2 \mathrm{D}}$ geschrieben werden als

$$
\mathbf{U}_{2 \mathrm{D}}=\mathbf{F}_{2 \mathrm{D}} \mathbf{F}_{2 \mathrm{D}} \text {. }
$$

Um dann die Quadratwurzel zu lösen, wird folgende spektrale Zerlegung verwendet:

$$
\mathbf{U}_{2 \mathrm{D}}=\lambda_{1} \mathbf{n}_{1} \mathbf{n}^{\mathrm{T}}{ }_{1}+\lambda_{2} \mathbf{n}_{2} \mathbf{n}^{\mathrm{T}}{ }_{2} .
$$

Hierbei sind $\lambda_{1}$ und $\lambda_{2}$ die Quadratwurzeln der Eigenwerte der symmetrischen Matrix $\mathrm{U}^{2} 2 \mathrm{D}$ und $\mathbf{n}_{1}$ und $\mathbf{n}_{2}$ die dazugehörigen Eigenvektoren. Die logarithmische (Hencky-) In-PlaneDehnung $\boldsymbol{\varepsilon}_{2 \mathrm{D}}$ ergibt sich schließlich zu [8]

$$
\boldsymbol{\varepsilon}_{2 \mathrm{D}}=\ln \left(\mathbf{U}_{2 \mathrm{D}}\right) .
$$

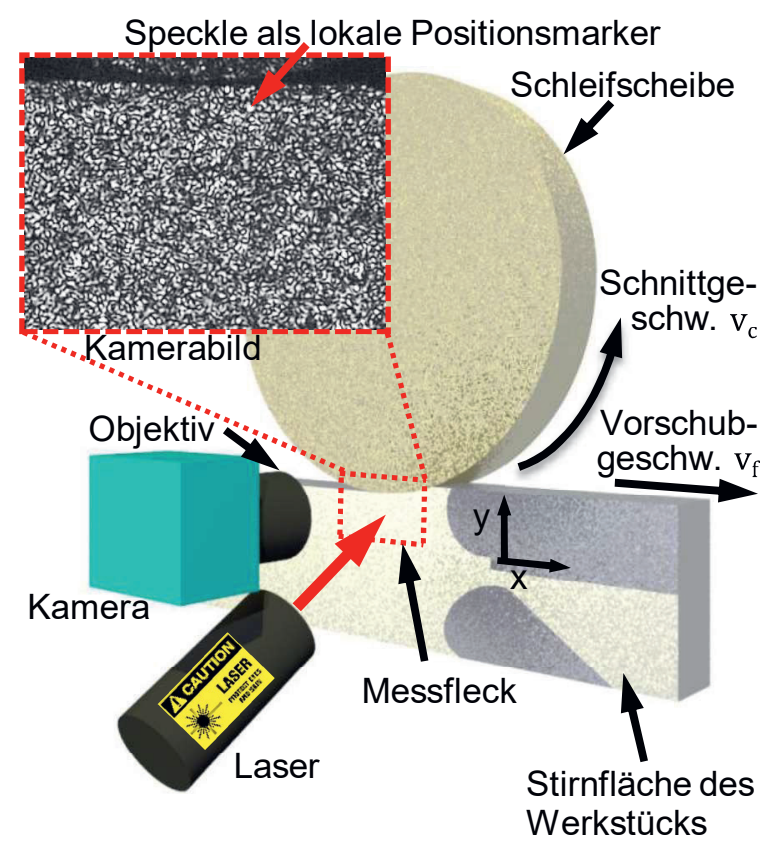

Abb. 1: Mess- und Einsatzprinzip der SpeckleFotografie beim Flachschleifen.

Das Grundprinzip für die Dehnungsmessungen im Flachschleifprozess ist in Abbildung 1 dargestellt. Das Messfeld befindet sich auf der Stirnfläche - der unbearbeiteten Seite des Werkstücks - direkt an der Grenze zur bearbeiteten Oberfläche. Das Objektiv und die CMOS-Kamera (complementary metal-oxidesemiconductor) mit globalen Shutter sind relativ zum Werkstücksystem fixiert, so dass auftretende Specklebewegungen direkt mit Verschiebungen von Oberflächenpunkten korreliert werden können.

Out-of-Plane-Verformungen der Oberfläche im Schleifprozess führen zu einer Dekorrelation des Specklemusters, wodurch der gewünschte Messeffekt zerstört wird. Der Einsatz einer Hochgeschwindigkeitskamera (Optronis: CP70) mit einer schnellen Bild-zu-Bild-Auswertung bei einer Bildrate von $167 \mathrm{fps}$ reduziert diese Dekorrelation, da die Out-of-Plane-Verschiebung in der kurzen Zeit von $1 / 167$ s so gering ist, dass fast keine Speckledekorrelation auftritt. 
Die gemessenen Verschiebungen in der Objektebene sind in dieser kurzen Zeit entsprechend klein. Mit einer geeigneten zweidimensionalen Sub-Pixel-Interpolation können jedoch Verschiebungen von wenigen Hundertstel Pixeln (hier 1 Pixel $\widehat{=} 1,94 \mu \mathrm{m}$ ) bestimmt werden [9].

\section{Experimenteller Aufbau}

Die Versuche wurden auf einer Blohm Profimat 412 HSG Profilschleifmaschine durchgeführt. Die Umsetzung des Messprinzips ist im unteren, vergrößerten Bereich von Abbildung $2 \mathrm{zu}$ sehen.

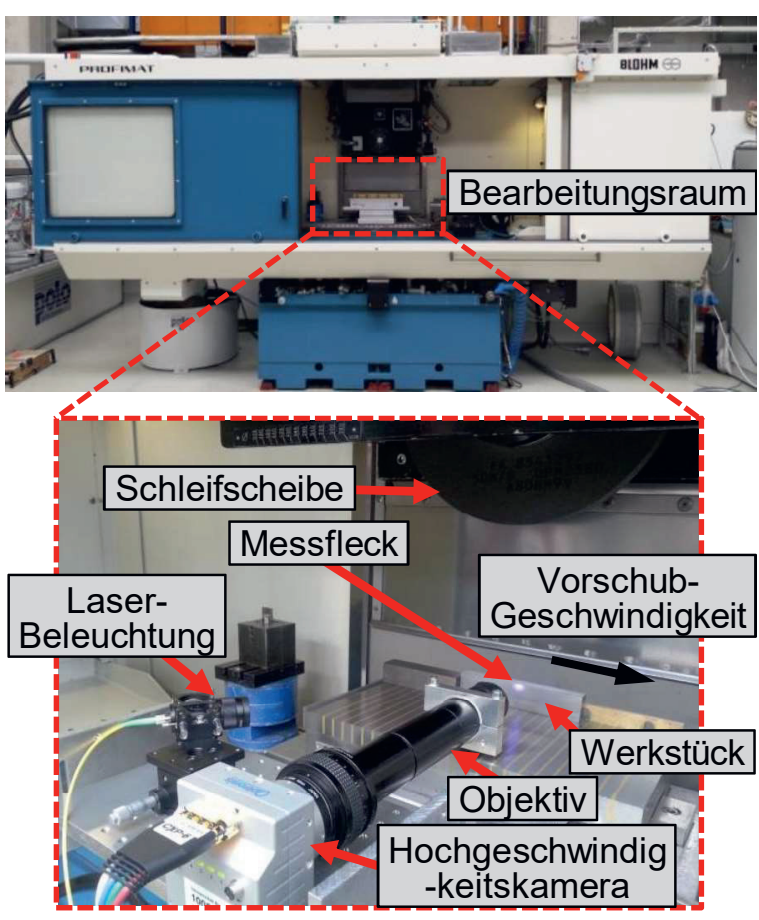

Abb. 2: Blohm Profimat 412 HSG Schleifmaschine und integrierter Messaufbau

Das Werkstück ist als Quader mit einer Länge von $165 \mathrm{~mm}, 18 \mathrm{~mm}$ Breite und 41,5 mm Höhe ausgeführt. Um die Peripheriegeräte (Kamera, Laser $(\lambda=405 \mathrm{~nm}, \mathrm{I}=80 \mathrm{~mW})$ und optische Komponenten) fest mit dem Messobjekt zu verbinden, werden die Adapter und Halterungen gemeinsam mit dem Werkstück magnetisch gespannt. Die dem Specklemesssystem zugewandte Werkstückseite wird vor den Experimenten kugelgestrahlt, um eine raue Oberfläche zu erzeugen, was zu einer ausreichenden Phasenmischung der Laserwellenfront und zur Bildung eines voll entwickelten und homogenen Specklemusters führt. Die Messung erfolgt an einer Stelle im mittleren Abschnitt des Werkstücks. Der Messaufbau ist mit der Linearbewegung des Werkstücks in $\mathrm{x}$ Achsrichtung verbunden. Daher bezieht sich die Vorschubgeschwindigkeit von $v_{f}=2 \mathrm{~m} / \mathrm{min}$ auf die relative Vorschubgeschwindigkeit der Schleifscheibe gegenüber dem Werkstück. Der Vorschub des Werkstücks ist gegenläufig zur Schnittgeschwindigkeit $\mathrm{v}_{c}=20 \mathrm{~m} / \mathrm{s}$ der Schleifscheibe. Somit bewegt sich die Vorschubgeschwindigkeit $\mathrm{v}_{\mathrm{f}}$ der Schleifscheibe in die gleiche Richtung wie $\mathrm{v}_{\mathrm{c}}$ (siehe Abbildung 1).

Ein sich verändernder Flüssigkeitsfilm auf der Oberfläche führt zu einer ständigen Speckledekorrelation, wodurch der eigentliche Messeffekt verschwindet. Um die In-Prozess-Fähigkeit der Speckle-Fotografie beim Flachschleifen zu demonstrieren, wird im ersten Ansatz deshalb ein trockener Schleifprozess betrachtet und auf den Einsatz von Kühlschmierstoff verzichtet.

Das Fehlen der Kühlschmierstoffe führt jedoch zu einer starken Entwicklung von Funkenflug (siehe Abbildung 3). Glühende Späne können die optischen Komponenten beschädigen, diese müssen deshalb abgedeckt oder in einem entsprechend großen Abstand vom Punkt des Werkzeugeingriffs positioniert werden. Der große Arbeitsabstand von ca. $90 \mathrm{~mm}$ wird mit einem Hochleistungs-Vergrößerungs-objektiv (Rodenstock Apo-Rodagon N) erreicht.

Der Einsatz verschiedener Tuben ermöglicht eine dreifache Vergrößerung und führt so zu einem Messfeld von ca. $6 \mathrm{~mm} \times 8 \mathrm{~mm}$. Um die Optik zu stabilisieren und eine feste Position der Kamera zum Werkstück beim Schleifen zu gewährleisten, wurde eine spezielle Montagevorrichtung entwickelt. Eine Fixierung am vorderen Ende des Objektivs verhindert die Übertragung von Vibrationen von der Maschine auf die Kamera (siehe Abbildung 2). Dadurch werden Schwingungen sowohl für die Messeinheiten als auch für das Werkstück ausgeglichen und es ist möglich, auch während des Schleifvorgangs scharfe Bilder des Objekts aufzunehmen.

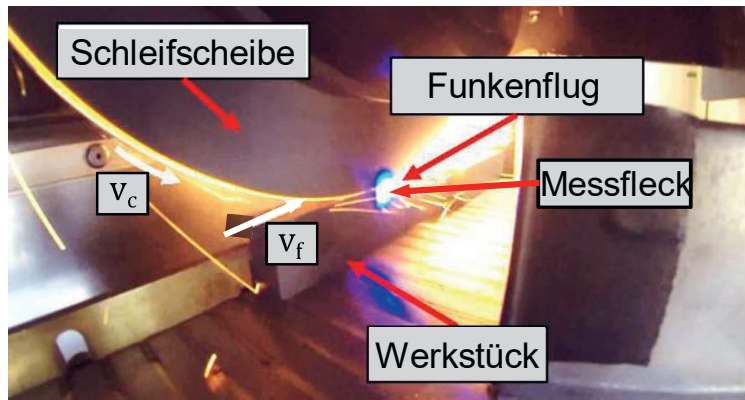

Abb. 3: Flachschleifprozess mit Funkenflug

Nach dem Schleifprozess konnte am Rand der bearbeiteten Oberfläche die Bildung eines Grats festgestellt werden. Die Gratbildung entspricht einer unterschiedlichen Neigung der Messfläche, wodurch das Licht aus dem oberen Teil des Werkstückbereichs nicht in die Kamera reflektiert wird. Dies führt zu einem 
Kontrastverlust des Specklemusters an der Oberkante des Werkstücks und damit zu einer hohen Messunsicherheit in diesem Bereich. Daher lässt sich das Verschiebungsfeld der oberflächennahen Kontaktzone nur bis zu einer Tiefe von ca. $800 \mu \mathrm{m}$ unterhalb der bearbeiteten Oberfläche berechnen. Unter Berücksichtigung der Schnitttiefe von $a_{e}=200 \mu \mathrm{m}$ wird der Bereich zwischen $\mathrm{y}=0 \mu \mathrm{m}$ und $\mathrm{y}=$ $1000 \mu \mathrm{m}$ unter der Oberfläche nicht bewertet (siehe Abbildung 4).

In den herkömmlichen Auswerteverfahren der Speckle-Fotografie wird der starke Partikelbzw. Funkenflug des trockenen Schleifprozesses als Verschiebung von Oberflächenpunkten fehlinterpretiert. Die Partikelbewegung kann durch den Einsatz optimierter Algorithmen allerdings nahezu vollständig herausgerechnet werden. Zu diesem Zweck wird die Größe der Speckle innerhalb des Auswertefensters mit der durchschnittlichen Specklegröße im globalen Specklebild verglichen. In der Regel sind störende Partikel durch Funkenbildung deutlich größer als der eingestellte Speckledurchmesser und somit sofort erkennbar. In diesem Fall ist die lokale Verformung für die Messposition auf null zu setzten. Der Fehler, der beim Summieren der Verschiebungen auftritt, ist aufgrund der hohen Taktfrequenz der Kamera vernachlässigbar.

Geht man von einem homogenen Werkstück aus, so kann durch die zeitliche Abfolge der Einzelbilder, der Messbereich in Bewegungsrichtung der Schleifscheibe ( $x$-Richtung) von $8 \mathrm{~mm}$ nahezu beliebig erweitert werden. In den gezeigten Messungen ist der Messbereich durch den Speicher der Kamera jedoch auf $32 \mathrm{~mm}$ beschränkt.

\section{Ergebnisse der In-Prozess-Messung für den Flachschleifprozess ohne Kühlschmierstoff}

Abbildung 4 zeigt die mittels SpeckleFotografie an der Stirnfläche des Werkstücks gemessenen Verformungsfelder in $x$ - und $y$ Richtung. Die tangentiale Kraftkomponente verschiebt das gesamte Werkstück um $10 \mu \mathrm{m}$ in Richtung der Werkzeugbewegung $\mathrm{v}_{\mathrm{c}}$. Die maximale $x$-Verschiebung liegt ca. $2,5 \mathrm{~mm}$ nach der ersten Interaktion zwischen Werkzeug und Werkstück und beträgt $22 \mu \mathrm{m}$ in Vorschubrichtung. Gegen Ende der Kontaktfläche zwischen Schleifscheibe und Werkstück verringert sich die $x$-Verschiebung auf ca. $15 \mu \mathrm{m}$.

Für die y-Komponente (Bild unten) ist eine Verschiebung nach unten, in negative $y$ Richtung, zu beobachten. Zehn Millimeter hinter der Schleifscheibe hat sich die Verschiebung in negative Richtung auf $25 \mu \mathrm{m}$ erhöht.
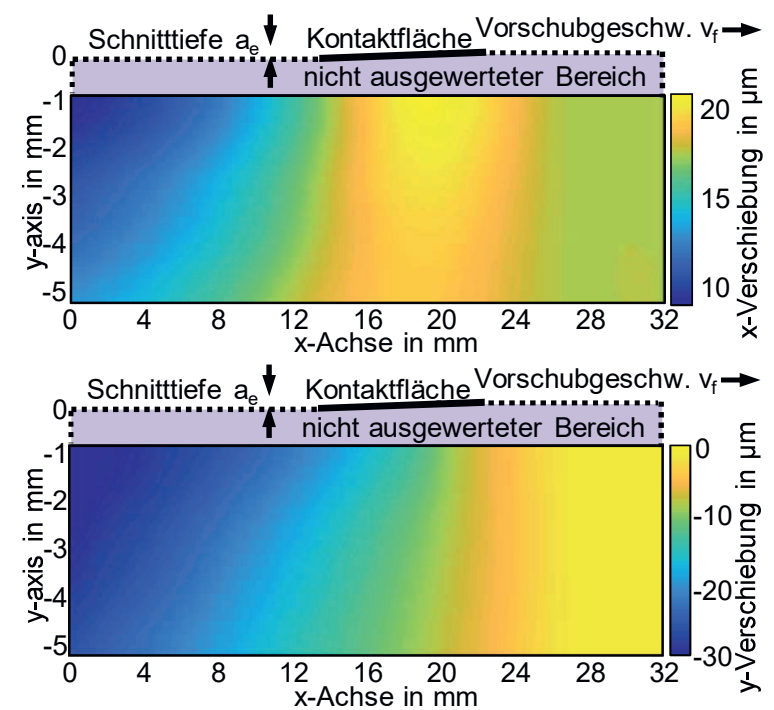

Abb. 4: Speckle-fotografisch gemessene Verschiebungsfelder, jeweils in $x$ - und $y$-Richtung

Die gemessenen Verschiebungen decken sich im Vergleich (bis auf $20 \%$ Abweichung) mit einem simulierten Schleifprozess bei gleichem Energieeintrag [10]. In der Simulation wurde der trockene Schleifprozess allerdings dadurch angenähert, dass sich eine konstante Wärmequelle über das Werkstück bewegt.

Aus den $x$ - und $y$-Verschiebungen wurde gemäß GIn. (1)-(6) die Dehnung in der Ebene der Stirnfläche des Werkstücks berechnet. Im oberen Teil von Abbildung 5 ist die Dehnung in x-Richtung aufgetragen. Das Minimum der $\mathrm{x}$-Dehnung liegt ungefähr 2-3 $\mathrm{mm}$ vor der Schleifscheibe und hat einen Wert von $-1,2 \times 10^{-3} \mathrm{~mm} / \mathrm{mm}$. Direkt hinter der Kontaktzone der Schleifscheibe ist das Maximum der $\mathrm{x}$-Dehnung $\mathrm{zu}$ finden, es besitzt einen Spitzenwert von $1,5 \times 10^{-3} \mathrm{~mm} / \mathrm{mm}$.
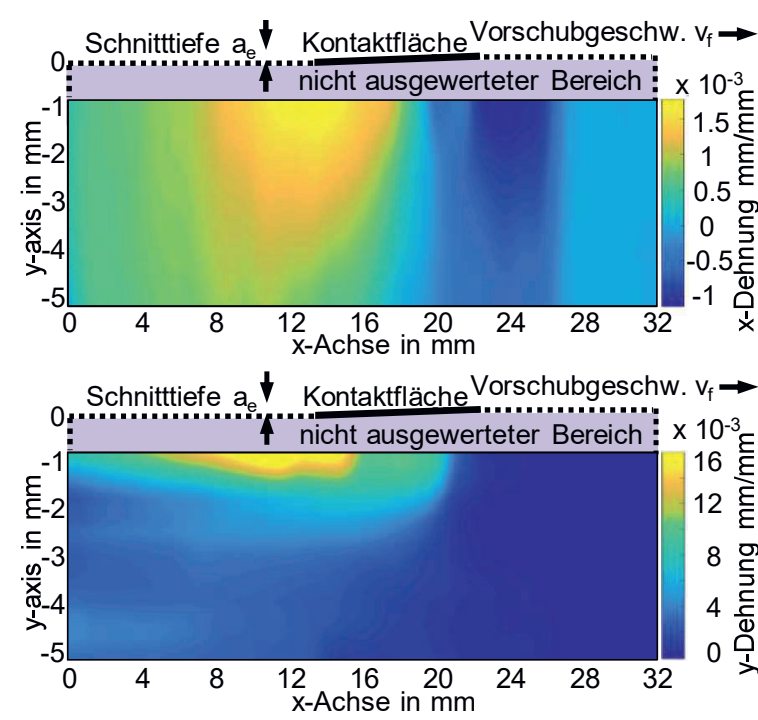

Abb. 5: Aus den entsprechenden Verschiebungsfeldern berechnete Dehnungen in $x$ - und y-Richtung 
Im unteren Teil von Abbildung 5 ist das Dehnungsfeld für die y-Richtung gezeigt. Das Maximum der y-Dehnung liegt am Ende der Kontaktfläche und steigt bis auf Werte von $16 \times 10^{-3} \mathrm{~mm} / \mathrm{mm}$ an.

Auch die gemessenen Dehnungen stehen mit einer Abweichung von weniger als $20 \%$ in guter Übereinstimmung zu den Simulationen der Literatur [10].

Die Speckle-Fotografie ist also hinreichend robust, um auch unter den anspruchsvollen Bedingungen des trockenen Flachschleifens mit starkem Funkenflug In-ProzessMessungen zu ermöglichen.

\section{Speckle-Fotografie-Messungen unter Ein- satz von Kühlschmierstoff}

Insbesondere der Einsatz unter realitätsnahen Bedingungen, wie bei der Verwendung von Kühlschmierstoffen, erfordert neue Mess- und Auswertekonzepte der Speckle-Fotografie. In der Regel werden in der Fertigungstechnik bearbeitete Oberflächen mit Kühl- oder Schmiermitteln kontaminiert. Auf mikroskopischer Ebene verändern sich die Schichtdicken dieser Kontaminationsfilme permanent, so dass selbst bei transparenten Fluiden keine statischen, stabilen Specklemuster zu beobachten sind. Kann die Variation der Schichtdicke verhindert werden, so lässt sich eine Dekorrelation der Speckle - zumindest bei transparenten, homogenen Fluiden - verhindern. Eine Auswertung mit Verformungs- und Dehnungsanalyse ist somit möglich.

Diesbezüglich zeigen erste Vorstudien, dass Messungen auch durch begrenzte Flüssigkeitsfilme möglich sind. In einem Laboraufbau (siehe Abbildung 6) kann die Probe variabel in eine Petrischale mit Kühlschmierstoff getaucht und die über den $\mathrm{x} / \mathrm{y}$-Versteller eingestellte Verschiebung von unten speckle-fotografisch gemessen werden. Selbst ein Schmierstofffilm von $h=1,5 \mathrm{~mm}$ Höhe führt beim Vergleich von eingestellter und gemessener Verschiebung lediglich zu Messabweichungen von unter 5\%. Voraussetzung ist allerdings eine begrenzende, transparente Fläche, durch die eine konstante Schichtdicke garantiert ist.

Die praktische Umsetzung dieses Laboraufbaus kann relativ einfach erfolgen, indem die Stirnfläche des Werkstücks durch eine Glasplatte abgedeckt und der Bearbeitungsprozess durch die Glasscheibe hindurch mit dem Messsystem beobachtet wird.

Der Spalt zwischen Glasplatte und Werkstück kann nach Abbildung 6 bis zu 1,5 mm betragen und sollte von unten kontinuierlich mit Kühlschmierstoff gespült werden. Partikel und Fremdkörper lassen sich so permanent mit dem Kühlschmierstoff noch oben aus dem
Spalt herausdrücken, wobei durch die über das Werkstück heraustretende Flüssigkeit gleichzeitig die Kühlung der Oberfläche erfolgt.

Prinzipiell ist die im trockenen Schleifprozess erprobte Speckle-Fotografie somit auch geeignet, um Fertigungsverfahren unter realitätsnahen Bedingungen $\mathrm{zu}$ untersuchen und somit einen Beitrag zum Verständnis der Prozesse, bzw. zum Aufstellen einer Prozesssignatur zu liefern.
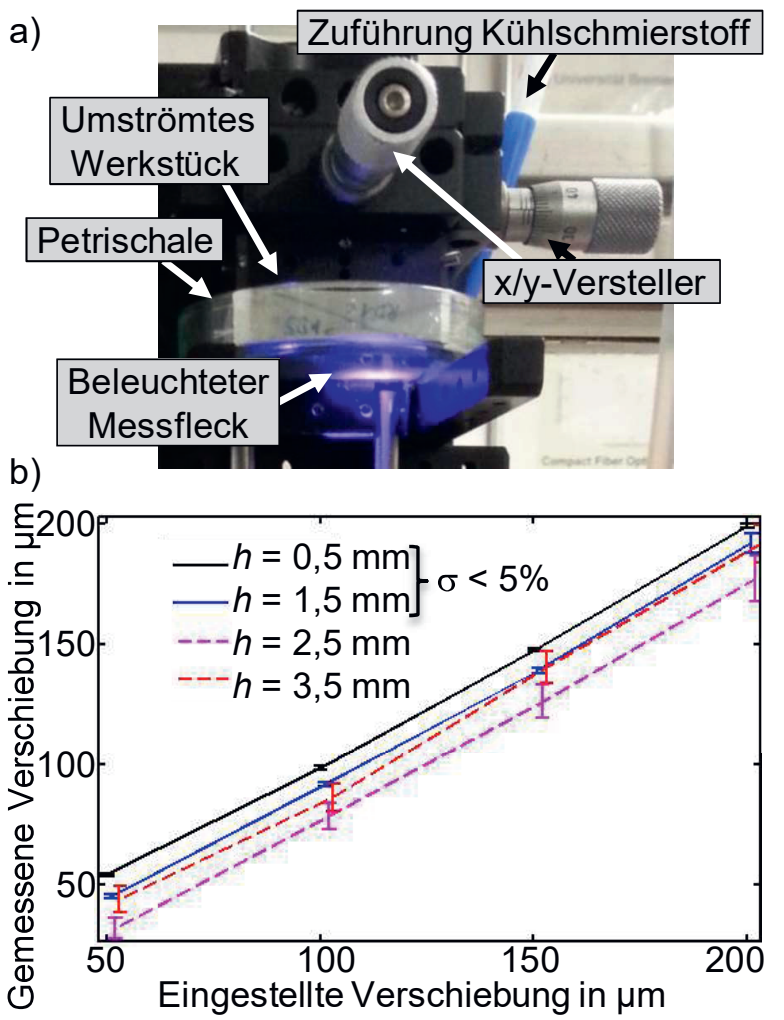

Abb. 6: Speckle-Fotografie-Messungen an einem in Öl getauchten Werkstück (a). Gemessene Verschiebung in Abhängigkeit vom eingestellten Wert und der Höhe des Flüssigkeitsfilms $h(b)$.

\section{Zusammenfassung und Ausblick}

In dem vorliegenden Beitrag werden während eines Schleifprozesses prozessbegleitend Verschiebungs- und Dehnungsfelder optisch gemessen. In dem zunächst betrachteten trockenen Schleifprozess ohne Kühlschmierstoff entsteht starker Funken- bzw. Partikelflug, welcher sich mit Schnittgeschwindigkeit durch das Sichtfeld des Messsystems bewegt. Trotz dieser anspruchsvollen Bedingungen mit störendem Funkenflug sind, mit entsprechend angepassten Auswerteverfahren der SpeckleFotografie, In-Prozess-Dehnungsmessungen möglich.

Die Dehnungsanalysen zeigen, dass der Energieeintrag sehr lokal erfolgt. Da die Ergebnisse nur um $20 \%$ von Simulationen unter Annahme 
einer konstant bewegten Wärmequelle [10] differieren, sind die eingebrachten Verformungen und Dehnungen im trockenen Schleifprozess offensichtlich in erster Linie thermischer Natur.

Labormessreihen durch einen begrenzten Kühlschmierstofffilm verdeutlichen den Einfluss der Schichtdicke des Fluids auf die SpeckleFotografie-Messung. Selbst bei begrenzten Schichtdicken von 1,5 mm steigt die Messabweichung auf lediglich 1,5\%. Die Ergebnisse geben somit Anlass, den Einsatz der SpeckleFotografie auch auf Fertigungsprozesse unter realitätsnahen Bedingungen und der Verwendung von Kühlschmierstoffen zu erweitern.

Daher wird aktuell ein Messaufbau für das Flachschleifen implementiert, bei dem der Spalt zwischen Werkstück und Messsystem bzw. einer begrenzenden Glasscheibe vollständig mit Kühlschmierstoff gefüllt ist.

Nach der erfolgreichen Messung der Beanspruchung und der im Werkstück verbleibenden Modifikation unter dem Einsatz von Kühlschmierstoffen, lässt sich die Prozesssignatur für dieses Fertigungsverfahren aufstellen.

Weiterhin kann das Messsystem zukünftig an die Kinematiken weiterer Fertigungsprozesse angepasst bzw. entsprechend erweitert werden, um einen Vergleich von verschiedener Fertigungsverfahren und eine Prozessübergreifende Beschreibung mittels Prozesssignatur zu realisieren.

\section{Danksagung}

Die Autoren danken der Deutschen Forschungsgemeinschaft (DFG) für die Förderung des Transregionalen Sonderforschungsbereichs SFB/TRR 136 "Prozesssignaturen", insbesondere des Teilprojekts C06. Sie danken auch Ewald Kohls (Teilprojekt F01) und seinen Kollegen aus dem Leibniz-Institut für Werkstofforientierte Technologien (IWT) für die freundliche Unterstützung bei der Durchführung der In-Prozess-Messungen.

\section{Literaturnachwe is}

[1] Archold, E.; Burch, J.M.; Ennos, A.E.: Recording of in-plane surface dis placements bydouble-exposure speckle photography. Opt. Acta $1970,17,12$, S. 883-898.

[2] Stetson, K.A.: A Review of Speckle Photography and Interferometry. Opt. Eng. 1975, 14, 5, S. $482-489$.

[3] Fischer, A.: Fundamental uncertaintylimit for speckle dis placement measurements. Applied Optics 2017, 56, S.7013-7019.

[4] Tausendfreund, A.; Stöbener, D.; Dumstorff, G.; Sarma, M.; Heinzel, C.; Lang, W.; Goch, G.: Systems for locally resolved measurements of physical loads in manufacturing processes. CIRP Annals - Manufacturing Technology2015, 64, 1, S. 495-498.

[5] Tausendfreund, A.; Stöbener, D.; Fischer, A.: Precise in-process strain measurements for the investigation of surface modification mechanisms. Journal of Manufacturing and Materials Processing 2018,2(1), 9 (11 S.).

[6] Zhou, P.; Goodson, K.E.: Subpixel displacementand deformation gradient measurement using digital image/s peckle correlation (DISC). Optical Engineering 2001, 40, 8, S. 1613-1620.

[7] Kajberg, J.; Lindkvist, G.: Characterisation of materials subjected to large strains byinverse modelling based on in-plane displacement fields. International Journal of Solids and Structures 2004, 41, S. 3439-3459.

[8] Eman, J.; Sundin, K.G.; Oldenburg, M.: Spatially resolved observations of strain fields at necking and fracture of anisotropic hardened steel sheet material. International Journal of Solids and Structures 2009, 46, S. 2750-2756.

[9] Tausendfreund, A.; Stöbener, D.; Goch, G.: Measuring technique for the detection of process force related component deformations in metalworking manufacturing processes. In: Zoch, H. W.; Lübben, T. (Hrsg.): 5th International Conference on Distortion Engineering (IDE), Bremen 2015, S. 355-364.

[10] Tausendfreund, A.; Borchers, F.; Kohls, E.; Kuschel, S.; Stöbener, D.; Heinzel, C.; Fischer, A.: Investigations on material loads during grinding by speckle photography. Journal of Manufacturing and Materials Processing 2018, 2(4), 71 (12 S.). 